\title{
A Capture Scenario for Globular Cluster $\omega$ Centauri
}

\author{
Toshio Tsuchiya $^{1}$, Dana I. Dinescu ${ }^{2,3}$, Vladimir I. Korchagin ${ }^{2,4}$
}

\begin{abstract}
We explore an accretion origin for $\omega$ Cen by N-body modeling of the orbital decay and disruption of a Milky-Way dwarf satellite. This work is focused on studying a particular satellite model that aims to reproduce the present orbit of $\omega$ Cen, as recently determined from absolute proper motions. The model satellite is launched from $58 \mathrm{kpc}$ from the Galactic Center, on a radial, low-inclination orbit. We find that a capture scenario can produce an $\omega$ Cen-like object with the current low-energy orbit of the cluster. Our best model is a nucleated dwarf galaxy with a Hernquist density profile that has a mass of $8 \times 10^{9} \mathrm{M}_{\odot}$, and a half-mass radius of $1.4 \mathrm{kpc}$.
\end{abstract}

Subject headings: (Galaxy:) globular clusters: individual(NGC 5139)

\section{Introduction}

Recent advances in understanding the nature of $\omega$ Centauri, the most massive Milky Way globular cluster $\left(5 \times 10^{6} \mathrm{M}_{\odot}\right.$, Meylan et al. 1995$)$, have now placed on a firmer ground its accretion origin as opposed to formation within the Milky Way. Unlike the majority of the Milky Way globular clusters, $\omega$ Cen is a complex chemical system with an extended star formation history. For instance, the multiple-peak metallicity distribution (e. g., Pancino et al. 2000, the correlation between age and metallicity (e.g., Hughes \& Wallerstein 2000), and the s-process heavy-element enhanced enrichment in cluster stars (e.g., Smith et al.

\footnotetext{
${ }^{1}$ Astronomishes Rechen-Institut, Mönchhofstraße 12-14, 69120 Heidelberg, Germany, e-mail: tsuchiya@ari.uni-heidelberg.de, and SGI Japan, Ltd. Kyoto city Skagura Bio-VIL 013, 668-3, MotoZaimokucho, Fushimi-ku, Kyoto, 612-8043 Japan

${ }^{2}$ Astronomy Department, Yale University, P.O. Box 208101, New Haven, CT 06520-8101, e-mail: dana@astro.yale.edu, vik@astro.yale.edu

${ }^{3}$ Astronomical Institute of the Romanian Academy, Str. Cutitul de Argint 5, RO-75212, Bucharest 28, Romania

${ }^{4}$ Institute of Physics, Stachki 194, Rostov-on-Don, Russia, 344090
} 
2000) argue for a star formation history of 2 to 4 Gyr. Furthermore, the correlation between the enhancement of s-process elements with metallicity argues for a self-enrichment process within this system (Vanture, Wallerstein \& Suntzeff 2002) as opposed to the build-up of the whole system by merging a few chemically distinct fragments. Gnedin et al. (2002) show that the present-day $\omega$ Cen is not unique among globular clusters in its ability to retain its own stellar ejecta, but its frequent passages through the Galactic disk should have swept out all of the s-process enriched gas from AGB stars. This is the case for all of the globular clusters within $10 \mathrm{kpc}$ of the Galactic center, but not for $\omega$ Cen.

The internal kinematics of $\omega$ Cen lend further support to an accretion origin. The cluster rotates relatively rapidly causing an ellipticity of $\sim 0.12$ (Merritt, Meylan, \& Mayor 1997), while most of the Galactic globular clusters have an ellipticity of $\sim 0.03$ (Majewski et al. 2001). However, the metal rich stars $([\mathrm{Fe} / \mathrm{H}] \geq-1.2)$ in $\omega$ Cen do not share the bulk rotation of the metal poor stars, and have a lower velocity dispersion (Norris et al. 1997). The metal rich stars also show a North-South elongation in contradistinction to the wellknown East-West elongation of most of $\omega$ Cen's stars (Pancino et al. 2000). The distinctive features of the metal-rich stars may be related to an accretion event within $\omega$ Cen.

The extended star-formation history of $\omega$ Cen, the presence of stars formed from AGBenriched gas, and the evidence of a merger/accretion event point to a formation of $\omega$ Cen not only away from the Milky Way, but also at the bottom of the potential well of a massive galaxy. Thus, we wish to explore a previously established hypothesis: $\omega$ Cen is the nucleus of a dwarf galaxy captured and disrupted by the Milky Way (Freeman 1993). A dwarf sinking to the center of the Galaxy is subject to dynamical friction with simultaneous mass loss due to the interaction with the Galactic tidal field. All of the dwarf is stripped off except for the nucleus which, presumably, is left with the present-day orbit of $\omega$ Cen. This orbit is retrograde, of low energy and low inclination, and highly eccentric. It has an apocenter of only $6.4 \mathrm{kpc}$, a pericenter of $\sim 1 \mathrm{kpc}$, and a period of $120 \mathrm{Myr}$ (Dinescu, Girard, \& van Altena 1999). Can the capture scenario produce the current orbit of $\omega$ Cen, and if so, under what conditions? Zhao (2002) made the first attempt to model this accretion event using a semi-analytical calculation. He found that, if the dwarf is launched from $50 \mathrm{kpc}$ from the Galactic center, the remnant will not decay to the present orbit. This is because strong tidal shocks reduce the mass to the point that dynamical friction becomes too inefficient to drag the system to the current low-energy orbit of $\omega$ Cen. Zhao's analysis was simplified by a number of assumptions. For instance, the mass outside the tidal radius is instantaneously stripped, and the bulge and disk contributions to the dynamical drag were not taken into account in the 50-kpc launch. We re-investigate the accretion scenario by means of N-body simulations in order to follow the evolution of a dwarf galaxy falling into the Milky Way. 


\section{The Models}

In our simulations, we use the model by Kuijken and Dubinski (1995) that reproduces closely an exact solution of the set of collisionless Boltzmann and Poisson equations. The parameters of the model have been determined based on the observed properties of the Milky Way. We assume the solar radius, the disk exponential scale length, and the disk vertical scale height to be $8 \mathrm{kpc}, 3.5 \mathrm{kpc}$ and $245 \mathrm{pc}$ respectively. The circular velocity of the disk at the solar radius is taken to be $220 \mathrm{~km} \mathrm{~s}^{-1}$. The surface density within $1.1 \mathrm{kpc}$ of the disk plane in the model is equal to $69.8 \mathrm{M}_{\odot} \mathrm{pc}^{-2}$ with the disk contribution being 45.5 $\mathrm{M}_{\odot} \mathrm{pc}^{-2}$. With the generally accepted cutoff radius of the disk of $28 \mathrm{kpc}$, the disk's total mass in the model is $5 \times 10^{10} \mathrm{M}_{\odot}$. The mass of the bulge is $0.75 \times 10^{10} \mathrm{M}_{\odot}$ within $2.4 \mathrm{kpc}$. The halo is approximated by a lowered isothermal sphere (Binney \& Tremaine 1987) with the central potential $\Psi(0) / \sigma_{0}^{2}=8$. The halo mass inside $50 \mathrm{kpc}$ is $4.9 \times 10^{11} \mathrm{M}_{\odot}$ which is within the observationally inferred limits $5.4_{-3.6}^{+0.2}$ (Wilkinson \& Evans 1999). A more detailed description of the Milky Way model can be found in Tsuchiya (2002).

We have examined the dynamics of the disrupting dwarf galaxy using two different density profiles: 1) a King profile, or lowered isothermal density distribution (King 1966), and 2) a Hernquist density profile (Hernquist 1990). Our King profile has a deep central potential $\left(\Psi(0) / \sigma_{0}^{2}=12\right)$ with a density distribution in the central regions equivalent to an $r^{-2}$ cusp within our numerical resolution. The highly-concentrated King profiles were chosen to model a satellite with a steep central density cusp in an attempt to mimic the nuclear region that would presumably end as an $\omega$ Cen-like object. For the King profile, we have examined a set of six models whose total masses and half-mass radii are summarized in Table 1 (K1 through K6). The Hernquist model has a shallower density distribution than the King model, with an $r^{-1}$ cusp in the central regions. We have examined nine models of the Henquist profile whose parameters are also listed in Table 1 (H1 through H9). In order to follow the dynamics of the central nucleus of a nucleated dwarf galaxy - as the hypothetical progenitor of $\omega$ Cen - we place at the center of our preferred model dwarf galaxy an extended 'particle' that has a mass of $10^{7} \mathrm{M}_{\odot}$, and $r_{1 / 2}=35 \mathrm{pc}$. We then follow the evolution of the dwarf galaxy and its central 'nucleus' until the nucleus sinks to an orbit close to the center of the Milky Way. A more detailed description of the simulations and results is given elsewhere (Tsuchia, Korchagin, \& Dinescu 2003).

The simulations have been done with the Self Consistent Field (SCF)-tree code that has a detailed description in Tsuchiya (2002). A hierarchical tree algorithm has been used to calculate the dynamics of the softened particles representing the disk, the bulge of the Milky Way, and the dynamics of the dwarf galaxy. We have used 70,000 particles to represent the disk, and 10,000 particles for the bulge. The results presented here make use of a rigid 
halo, to avoid a long computational time; these models did not take into account dynamical friction on the halo. We have however tested one model (i.e., our preferred one, H4, see Table 1 , and the following Section) using a "living" halo. This halo has been modeled with the SCF algorithm (Hernquist \& Ostriker 1992). The halo distribution is sampled by 100,000 particles. We have taken the tolerance parameter to be 0.7 , and the softening length to be 0.01 of the disk scale length which corresponds to $35 \mathrm{pc}$ in our Milky Way model. We found that the decay time for the simulation with the living halo is shorter than that for the rigid one (1.8 Gyr versus $3 \mathrm{Gyr}$ ), but the relation between the satellite's bound mass and apocenter distance is similar for the two halos (Tsuchia, Korchagin, \& Dinescu 2003).

The King model dwarfs are sampled by 50,000 equal-mass particles. For the Hernquist model dwarfs, we adopt a multi-mass N-body model. This is because we need to achieve a better mass resolution in the central regions of the dwarf galaxy, as the Hernquist model undergoes heavy mass loss down to $\sim 10^{7} \mathrm{M}_{\odot}$, while the King models stop losing mass at $\sim 10^{8} \mathrm{M}_{\odot}$ (see the next Section). The Hernquist distribution function has been divided into three energy groups. The lowest energy group is sampled by 50,000 particles with the mass of each particle being $2 \times 10^{-7}$ of the total mass of the dwarf. The middle and the highest energy groups are sampled by particles with masses $2 \times 10^{-6}$, and $2 \times 10^{-5}$ of the total mass of the dwarf, respectively, and the number of particles in each group is 45,000. The dwarf was hence sampled by 140,000 particles in total. The multi-mass model allows us to achieve a much better resolution of the dynamics of the central regions of the dwarf compared to that given by equal mass models. The dynamics of the central $10^{7} \mathrm{M}_{\odot}$ of the dwarf is therefore sampled by approximately 50,000 particles.

\section{The Sinking Satellite}

To approximately match the current orbit of $\omega$ Cen, we have chosen the initial position and velocity of the satellite to be $(\mathrm{X}, \mathrm{Y}, \mathrm{Z})=(50,0,30) \mathrm{kpc}$, and $\left(V_{X}, V_{Y}, V_{Z}\right)=(0,-20$, $0) \mathrm{km} \mathrm{s}^{-1}$. Here, $\mathrm{X}$ is positive away from the Galactic center, $\mathrm{Y}$ is positive toward Galactic rotation, and $\mathrm{Z}$ is positive toward the north Galactic pole; the Sun is at $(\mathrm{X}, \mathrm{Y}, \mathrm{Z})=(8$, $0,0)$. Due to the small initial velocity, the dwarf falls into a very eccentric orbit with a typical pericenter of $\sim 1 \mathrm{kpc}$. At each pericentric passage, the dwarf experiences strong shocks mainly from the encounters with the bulge which strip off the dwarf's outer layers. Simultaneously with the mass loss, the dwarf sinks into a smaller orbit.

The King and the Hernquist models have qualitatively different evolutionary sequences. In Figure 1 we show the bound mass (at apocenter) as a function of the apocentric distance for the King profiles (Fig. 1 a) and for the Hernquist profiles (Fig 1 b). For the same total 
mass, the King models with higher central densities (Table 1) sink deeper. Once the dwarf mass decreases to $\sim 10^{8} \mathrm{M}_{\odot}$, there is practically no more orbital decay. These models lose mass approximately exponentially with time. The ever decreasing mass-loss rate requires a time that is longer than the age of the Galaxy in order to reach a remnant with a bound mass of $\sim 10^{7} \mathrm{M}_{\odot}$ (Tsuchiya, Korchagin \& Dinescu 2003). For the Hernquist model dwarfs the evolutionary sequences (Fig. 1 b) show that the orbit decay stops at a given radius that depends on the central density (see Table 1). The dwarf continues to lose significant mass as opposed to the King models. In fact, all the models but H1 have completely dissolved after a time less than 5 Gyr. From these tests, we have chosen as our best representation of the progenitor of $\omega$ Cen, model H4. In Figure 2 we show the galactocentric distance of the center of mass of the tidally bound mass of the disrupting dwarf as a function of time for the model that includes the nucleus, and for the model that doesn't (see Section 2). After approximately 2 Gyr from the beginning of the simulations, the model with the nucleus establishes itself into a low-energy orbit with pericentric and apocentric distances of about $1 \mathrm{kpc}$ and $6 \mathrm{kpc}$. The time evolution of the galactocentric radius of the nucleated model illustrates that indeed the nucleus has near-stationary radial oscillations after 2.5 Gyr (Fig. 2 ). The non-nucleated model is completely disrupted in 2 Gyr. This is more clearly seen in Figure 3, where the mass of the gravitationally bound particles is shown as a function of time for the nucleated model, and the non-nucleated one. After 3 Gyr from the beginning of the simulation, the mass of the gravitationally bound particles surrounding the central nucleus is about $2 \times 10^{7} \mathrm{M}_{\odot}$ as can be seen in Figure 3. By 4 Gyr, further stripping decreases the mass of the gravitationally bound mass of the nucleated dwarf to the mass of the central nucleus. We speculate that this is a likely evolutionary history of the progenitor of $\omega$ Cen.

In Figure 4 we show a snapshot of the distribution of the stripped material taken at 3 Gyr. The left panel shows the distribution in the Galactic plane, while the right panels shows the distribution perpendicular to the Galactic plane. The stripped particles fill in a flattened, disk-like volume with a radius of about $10 \mathrm{kpc}$, and a vertical scale height of about $2.5 \mathrm{kpc}$. The volume density of the stripped matter in the solar neighborhood is low however. The estimated volume density of the stripped matter at $R \sim 8 \mathrm{kpc}$ is about 2 $\times 10^{-5} \mathrm{M}_{\odot} \mathrm{pc}^{-3}$. Dinescu (2002) analyzed the Beers et al. (2000) kinematically unbiased catalog of metal poor stars, and found an excess of RR Lyrae stars in the retrograde sample when compared to the prograde sample. The metallicity range of the sample where this excess was found is $[\mathrm{Fe} / \mathrm{H}]=-2.0$ to -1.5 , and the orbital eccentricities are larger than 0.8 (see Fig. 3 in Dinescu 2002). These properties are representative of $\omega$ Cen and its debris characteristics. Specifically, $30 \mathrm{RR}$ Lyrae stars were found in the retrograde sample, and 15 in the prograde one, amounting to an excess of 15 stars within a volume of $175 \mathrm{kpc}^{3}$. Cseresnjes (2001) estimated a population of $\sim 8400$ RR Lyrae stars in the Sagittarius dwarf 
spheroidal galaxy (Sgr), assuming that the galaxy lost about $50 \%$ of its total mass. Assuming also that RR Lyrae stars trace the light, he estimated an integrated absolute V-magnitude of -14.7 for the whole Sgr system. Estimating the number of RR Lyrae stars in a dwarf galaxy from the integrated absolute $\mathrm{V}$-magnitude, assuming a mass-to-light ratio $\mathrm{M} / \mathrm{L}_{V}$ of 7 (e. g., Prada \& Burkert 2002), and using the density of debris in the solar neighborhood estimated by our preferred model, we obtain a number of $65 \mathrm{RR}$ Lyrae stars in the volume of $175 \mathrm{kpc}^{3}$. Keeping in mind that the Beers (2000) catalog is not complete, the number of excess RR Lyrae found by Dinescu (2002) is in reasonable agreement with the expected number from $\omega$ Cen progenitor's debris.

\section{Summary}

We have shown that a dwarf satellite with an initial mass of $8 \times 10^{9} \mathrm{M}_{\odot}$, a Hernquist density profile of $r_{1 / 2}=1.4 \mathrm{kpc}$, and a nucleus of $10^{7} \mathrm{M}_{\odot}$ can be dragged to the current lowenergy orbit of $\omega$ Cen. The remnant is a central nucleus with a mass within a factor of 2 to a few of that of present-day $\omega$ Cen. We suggest two refinements to the model to fully reconcile the current mass of $\omega$ Cen with that of the remnant. The first one is purely computational in nature: one can explore results from higher-resolution simulations that allow a $10^{6} \mathrm{M}_{\odot}$ nucleus. The second one involves a more sophisticated model: one can replace the nucleus with a collection of individual particles in order to follow the mass loss due to frequent disk passages, presumably down to the present-day mass of $\omega$ Cen. In fact, Leon, Meylan \& Combes (2000) show that $\omega$ Cen has tidal tails related to its last passage through the disk, and indicating mass loss.

This study lends support to the hypothesis that $\omega$ Cen is the core of a disrupted satellite galaxy captured from the outer halo, and it underlines that the dwarf needs specific initial conditions in order to reproduce $\omega$ Cen's characteristics. Our results do not exclude the scenario proposed by Zhao (2002) where the satellite is captured from the edge of the Galactic disk. This latter scenario however, implies ram-pressure stripping by the Galactic disk, thus challenging the complex abundance pattern that $\omega$ Cen possesses. The debris from the parent galaxy forms a disk-like structure within $6 \mathrm{kpc}$ of the Galactic center. Kinematical surveys within 1-2 kpc of the Sun should be able to detect such a structure, as a retrograde feature (Dinescu 2002).

We thank T. Girard for many useful suggestions concerning the calculations and W. van Altena for his comments on the manuscript. This research was supported in part by the NSF under grant AST 0098687. VIK acknowledges R. Spurzem for providing partial support at ARI, Heidelberg through the DFG grant RUS 17/112/02. 


\section{REFERENCES}

Beers, T. C., Chiba, M., Yoshii, Y., Platais, I., Fuchs, B., \& Rossi, S. 2000, AJ, 119, 2866

Binney, J., \& Tremaine, S. 1987, Galactic Dynamics. Princeton Univ. Press, Princeton, NJ Cseresnjes, P. 2001, A\&A, 375, 909

Dinescu, D. I. 2002, in " $\omega$ Centauri A Unique Window into Astrophysics", ASP Conf. Ser., vol. 265, ed. F. van Leeuwen, J. D. Hughes, and G. Piotto, p. 365

Dinescu, D. I., Girard, T. M., \& van Altena, W. F. 1999, AJ, 117, 1792

Freeman, K. 1993, in IAU Symp. 153, Galactic Bulges, ed. H. Dejonghe and H.J. Habing (Dordrecht: Kluwer), p. 263

Gnedin, O. Y., Zhao, H. S., Pringle, J. E., Fall, S. M., Livio, M., \& Meylan, G. 2002, ApJ, 568,23

Hernquist, L., 1990, ApJ, 356, 359

Hernquist, L., \& Ostriker, J. P. 1992, ApJ, 386, 375

Hughes, J., \& Wallerstein, G. 2000, AJ, 119, 1225

King, I. 1966, AJ, 71, 64

Kuijken, K., \& Dubinski, J. 1995, MNRAS, 277, 1341

Leon, S., Meylan, G. \& Combes, F. 2000, A\&A, 358, 907

Majewski, S. R., Patterson, R. J, Dinescu, D. I., Johnson, W. Y., Ostheimer, J. C., Kunkel, W. E., \& Palma, C. 2000, in "The Galactic Halo: from Globular Clusters to Field Stars", Proc. of the 35th Liege Colloq., ed A. Noels, P. Magin, D. Caro, E. Jehnin, G. Parmentier, \& A. A. Thoul, p. 619

Merritt, D, Meylan, G., \& Mayor, M. 1997, AJ, 114, 1074

Meylan, G., Mayor, M., Duquennoy, A. \& Dubath, P. 1995, A\&A, 303, 761

Norris, J. E., Freeman, K. C., Mayor, M., \& Seitzer, P. 1997, ApJ, 487, 187

Pancino, E., Ferraro, F. R., Bellazzini, M., Piotto, G., \& Zoccali, M. 2000, ApJ, 534, 83

Prada, F., \& Burkert, A. 2002, ApJ, 564, L73

Smith, V. V., Suntzeff, N. B., Cuhna, K., Gallino, R., Busso, M., Lambert, D. L., \& Straniero, O. 2000, AJ, 119, 1239

Tsuchiya, T. 2002, New Astron., 7, 293

Tsuchiya, T., Korchagin, V. \& Dinescu, D. I. 2003, in preparation

Vanture, A. D., Wallerstein, G. \& Suntzeff, N. B. 2002 ApJ, 569, 984 
Wilkinson, M.I., \& Evans N.W. 1999, MNRAS, 310, 645

Zhao, H. S. 2002, in " $\omega$ Centauri A Unique Window into Astrophysics", ASP Conf. Ser., vol. 265, ed. F. van Leeuwen, J. D. Hughes, and G. Piotto, p. 391

This preprint was prepared with the AAS IATEX macros v5.0. 
Fig. 1.- Bound mass versus apocentric distance diagram showing the evolution of a sinking dwarf satellite. Panel a) shows the King model dwarfs, while panel b) the Hernquist model dwarfs.

Fig. 2. - The time dependence of the radius of a sinking dwarf satellite: nucleated - $\mathrm{x}$ symbols, non-nucleated - cross symbols. The inset box shows the time dependence for the radius between 2.8 and 3 Gyr.

Fig. 3. - The bound mass of the dwarf as a function of time. Symbols are the same as in Fig. 2. The non-nucleated dwarf is completely disrupted in 2 Gyr. The bound mass of the nucleated dwarf approaches a mass of about $10^{7} \mathrm{M}_{\odot}$ after $3 \mathrm{Gyr}$ of evolution.

Fig. 4.- A snapshot of the stripped particles taken at 3 Gyr. The left panel shows the distribution in the Galactic plane, while the right panel, that perpendicular to the Galactic plane. 
Table 1. Model Parameters

\begin{tabular}{ccc}
\hline \hline & & \\
Model & $M_{\text {tot }}\left(M_{\odot}\right)$ & $r_{1 / 2}(\mathrm{kpc})$ \\
\hline & & \\
K1 & $4 \times 10^{9}$ & 1.0 \\
K2 & $4 \times 10^{9}$ & 2.0 \\
K3 & $4 \times 10^{9}$ & 4.0 \\
K4 & $8 \times 10^{9}$ & 1.0 \\
K5 & $8 \times 10^{9}$ & 2.0 \\
K6 & $8 \times 10^{9}$ & 4.0 \\
& & \\
H1 & $4 \times 10^{9}$ & 1.0 \\
H2 & $4 \times 10^{9}$ & 2.0 \\
H3 & $4 \times 10^{9}$ & 4.0 \\
H4 & $8 \times 10^{9}$ & 1.414 \\
H5 & $8 \times 10^{9}$ & 2.282 \\
H6 & $8 \times 10^{9}$ & 5.657 \\
H7 & $16 \times 10^{9}$ & 2.0 \\
H8 & $16 \times 10^{9}$ & 4.0 \\
H9 & $16 \times 10^{9}$ & 8.0 \\
\hline
\end{tabular}




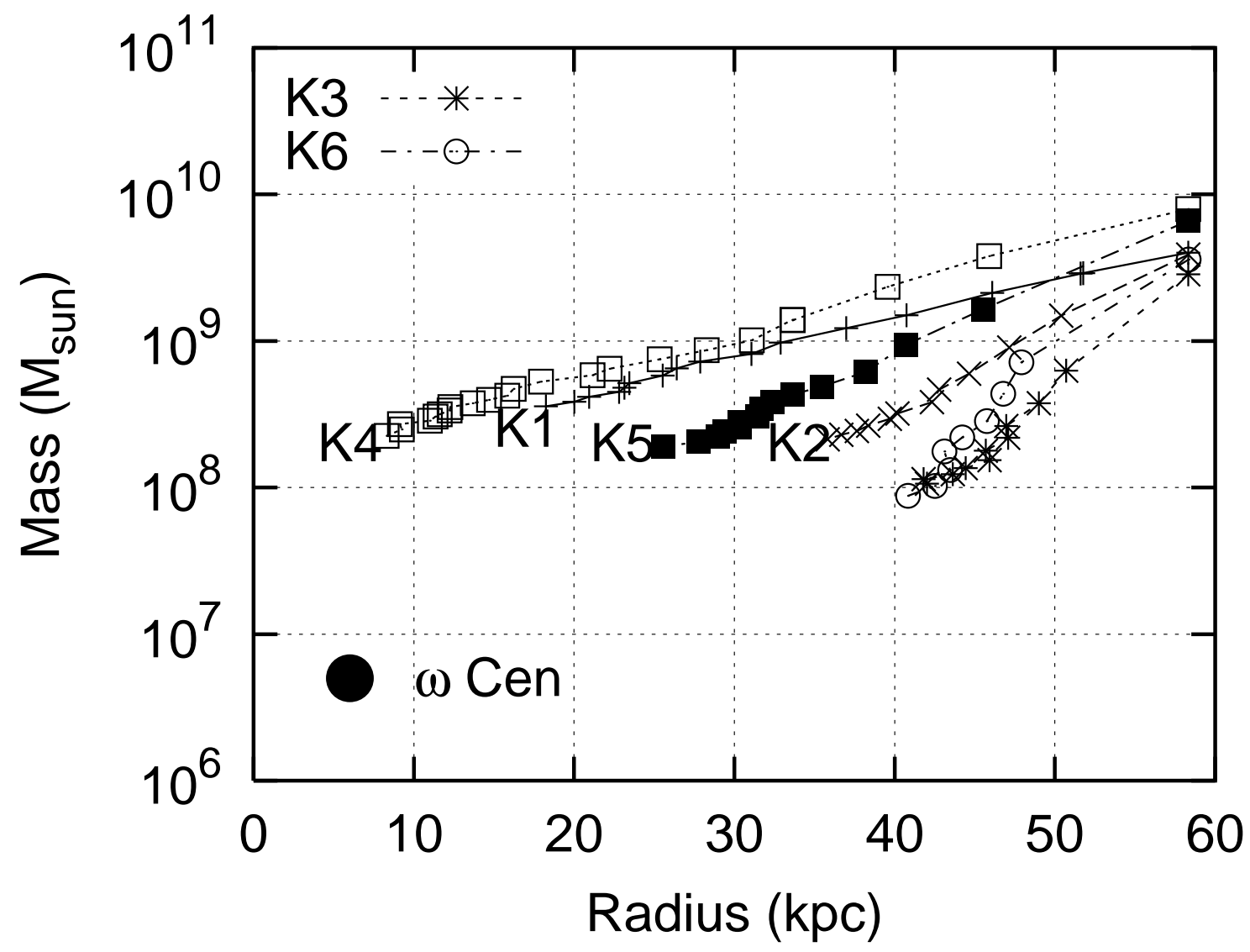




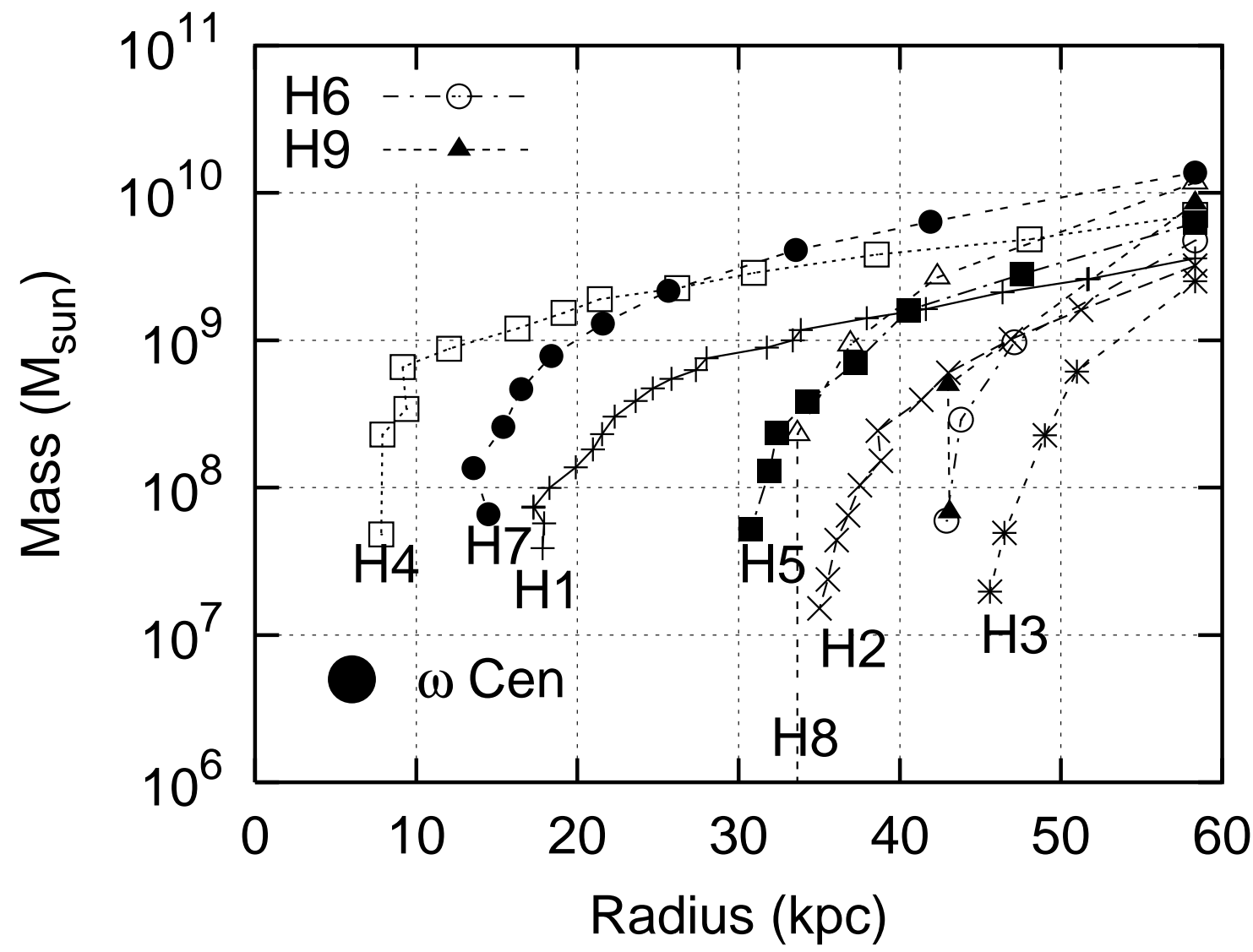




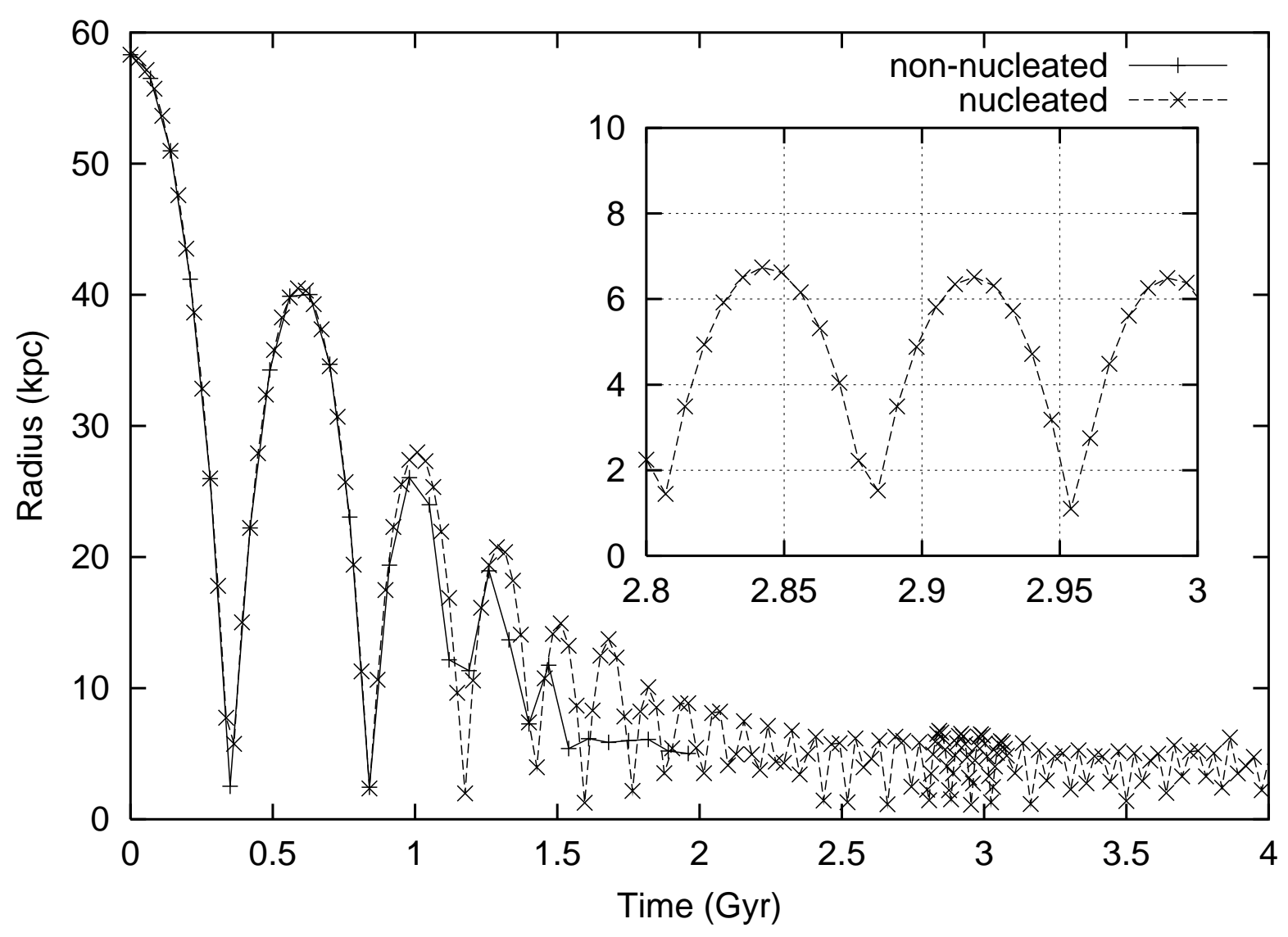




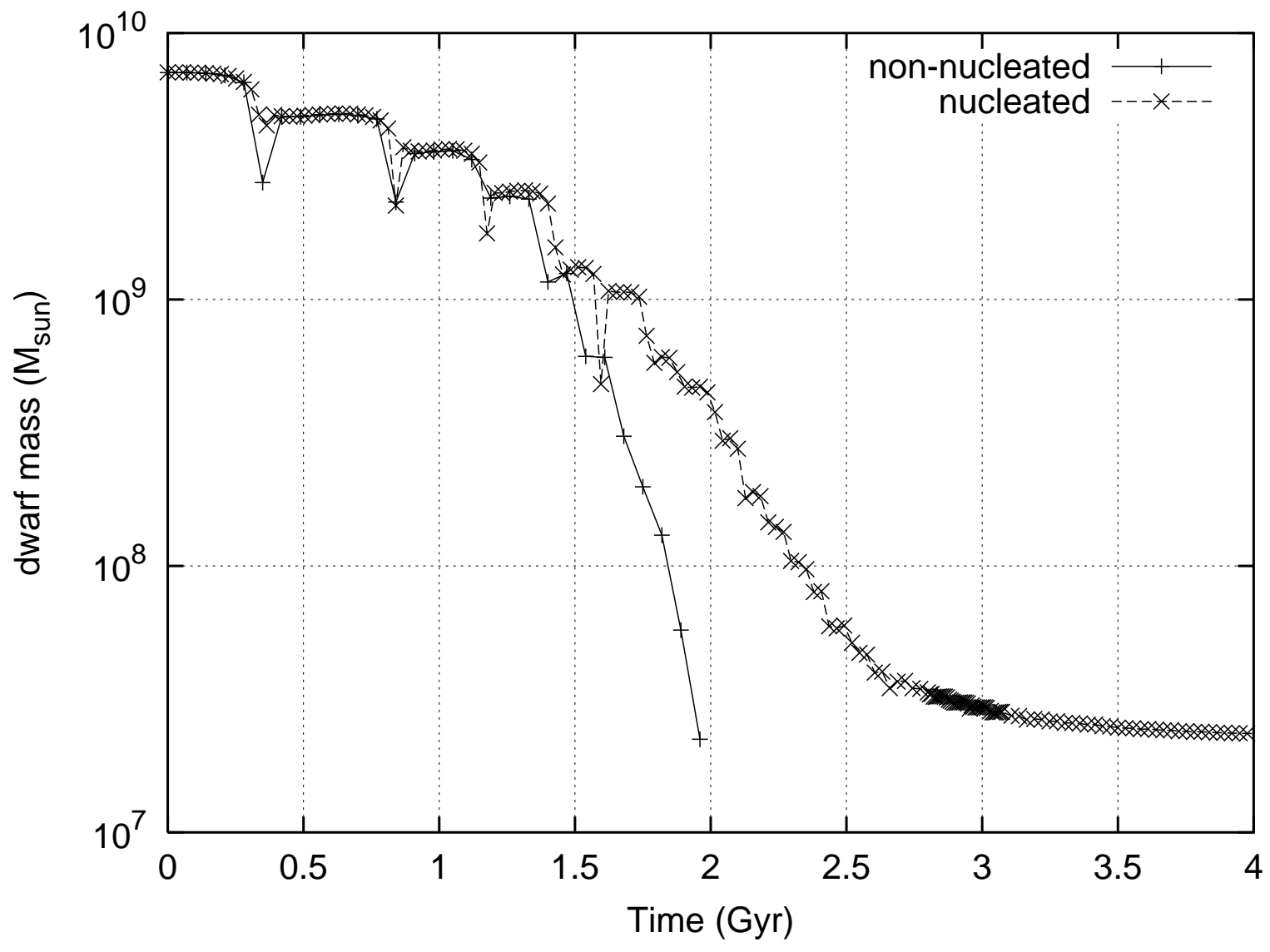



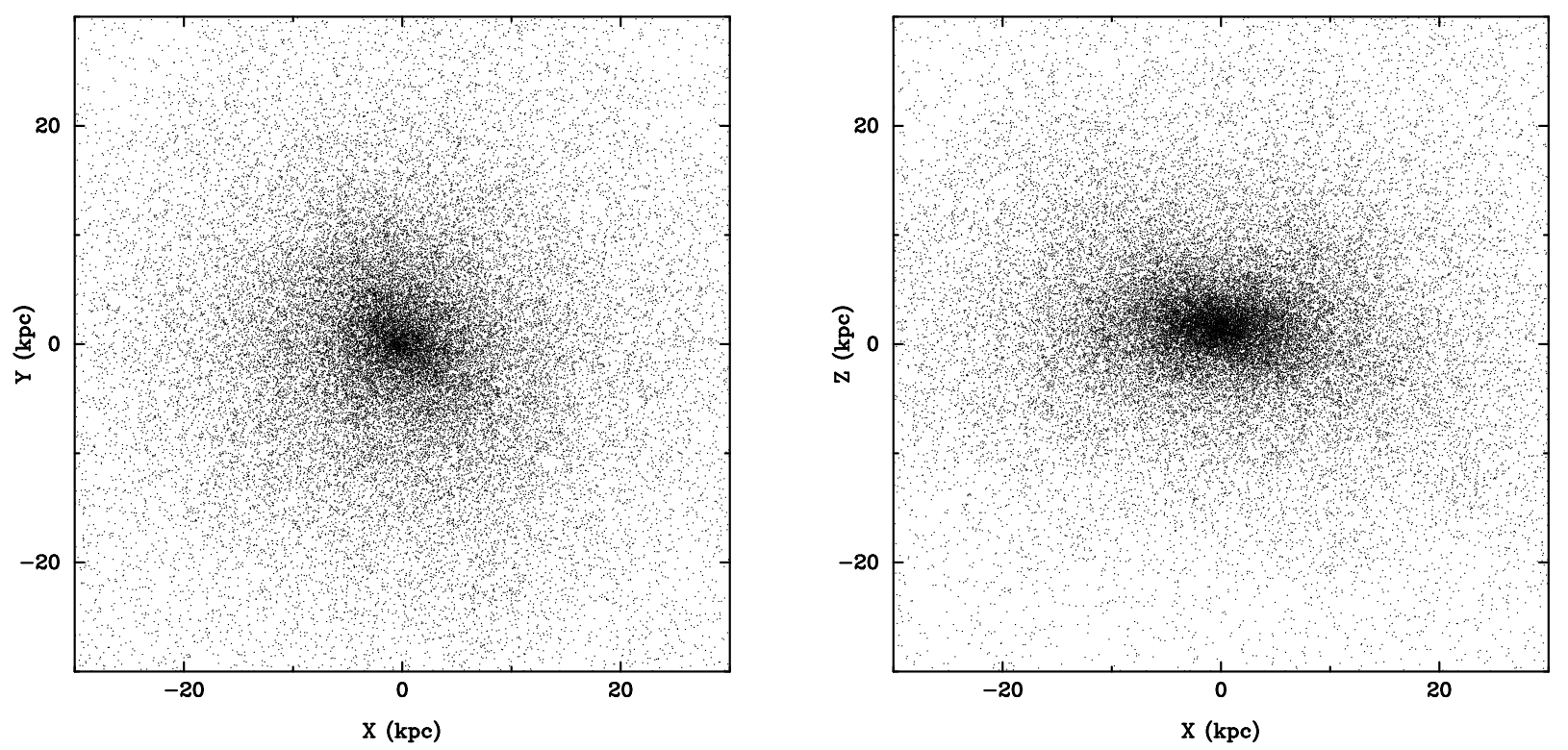